\title{
STUDY OF $\mathrm{VO}_{2}$ MAX DURING PHASES OF MENSTRUATION IN YOUNG FEMALE ATHLETES
}

Sunitha. G1 ${ }^{1}$ Ravi. B.N², Sudhir. G.K³.

1. Assistant Professor, Department of Physiology, Adichunchanagiri institute of medical sciences, B.G.Nagara, Nagamangala Taluk, Mandya District, Karnataka.

2. Post Graduate student, Department of Physiology, Adichunchanagiri institute of medical sciences, B.G.Nagara, Nagamangala Taluk, Mandya District, Karnataka.

3. Associate Professor, Department of Physiology, Adichunchanagiri institute of medical sciences, B.G.Nagara, Nagamangala Taluk, Mandya District, Karnataka.

\author{
CORRESPONDING AUTHOR \\ Dr. Sunitha. G, \\ Assistant Professor, \\ Adichunchanagiri institute of medical sciences, \\ B.G. Nagara, Nagamangala Taluk, Mandya, \\ District, Karnataka- 571448. \\ Email-drsuni1980@yahoo.co.in
}

HOW TO CITE THIS ARTICLE:

Sunitha. G, Ravi. B.N, Sudhir G.K. "Study of $\mathrm{VO}_{2}$ Max during Phases of Menstruation in Young Female Athletes". Journal of Evolution of Medical and Dental Sciences 2013; Vol2, Issue 23, June 10; Page: 4070-4078.

ABSTRACT: Context: The menstrual cycle is a natural monthly event in young women, coordinated by the hypothalamic-pituitary ovarian axis and may influence physiological and pathological changes that occur throughout the life time of the woman. There is a cyclical change in sex hormones during the course of menstrual cycle that have the potential effects on exercise capacity and performance through numerous mechanisms, such as substrate metabolism, cardiorespiratory function, thermoregulation, psychological factors and injuries. Consequently hormone level changes may theoretically lead to either improved or decreased performance at various times throughout the menstrual cycle. Physiological indices such as heart rate, tension, $\mathrm{VO}_{2}$ max and aerobic power are of the main indicators of exercise performance, a potential change in these indices over the menstrual cycle will have large practical implications for female athletes. $\mathrm{VO}_{2}$ max is highly predictive of endurance performance, hence the present study was done to evaluate whether there is any changes in the $\mathrm{VO}_{2}$ max during phases of menstruation in young female athletes. Aim: The present study was done to evaluate the $\mathrm{VO}_{2}$ max during phases of menstruation in young female athletes. Settings and Design: The present study was a cross sectional study consisting of 30 healthy female athletes of 17-20 years of age. Materials and Methods: This study was conducted in the Department of Physiology, Adichunchanagiri institute of medical sciences after the institutional ethical clearance and written consent from each participant. $\mathrm{VO}_{2}$ max (maximum oxygen uptake) was indirectly assessed by the Astrand nomogram method from submaximal exercise data obtained using Harvard step test during all the three phases of menstrual cycle. Results: The parameters were analyzed for statistical significance using Students' $t$ ' test and $p<0.05$ was considered the level of significance. There were no significant changes in the $\mathrm{VO}_{2}$ max during all the phases of menstrual 
cycle. Conclusions: This study showed that $\mathrm{VO}_{2}$ max is not significantly affected by the changing sex steroid levels observed across the normal menstrual cycle.

KEYWORDS: $\mathrm{VO}_{2}$ max, astrand nomogram, female athlete

INTRODUCTION: The female athlete has a unique physiology and a kaleidoscope of changing hormonal profiles throughout her biological life cycle. Patterns of male and female sex steroids after the onset of puberty are largely responsible for gender differences in morphology, aerobic and anaerobic capacity, strength and bone density, although socio-cultural influences cannot be ignored. In studies that compare male and female athletes, it also is critical that the subjects are not only matched for initial fitness levels, but also exercised at a comparable proportion of their maximal aerobic fitness. Nevertheless, the relative health benefits of physical activity and training in girls and women are similar to those of their male counterparts (1). It then becomes of interest, particularly for competitive female athletes in the reproductive age group, to consider potential differential effects of these hormones in the context of athletic performance.(2)

Aerobic power, aerobic capacity and maximal oxygen uptake are all terms used interchangeably with $\mathrm{VO}_{2}$ max.

$\mathrm{VO}_{2}$ max is the maximum capacity to transport and utilize oxygen during incremental exercise (Harvard step test) (The derivation is $\mathrm{V}$ - volume per time, $\mathrm{O}_{2}$ - oxygen, max - maximum). It is also called maximum oxygen consumption or maximal oxygen uptake.(3) Expressed either as an absolute rate in litres of oxygen per minute $(\mathrm{l} / \mathrm{min})$ or as a relative rate in milliliters of oxygen per kilogram of bodyweight per minute $(\mathrm{ml} / \mathrm{kg} / \mathrm{min})$, the latter expression is often used to compare the performance of endurance sports athletes. It is also known as aerobic capacity, which reflects physical fitness of a person. Because oxygen consumption is linearly related to energy expenditure, when we measure oxygen consumption, we are indirectly measuring an individual's maximal capacity to do work aerobically.

Numerous studies show that one can increase $\mathrm{VO}_{2}$ max by working out at an intensity that raises heart rate to between 65 and $85 \%$ of its maximum for atleast 20 minutes three to five times a week. A mean value of $\mathrm{VO}_{2}$ max for male athletes is about 3.5 litres/minute and for female athletes it is about 2.7 litres/minute.

As exercise intensity increases so does oxygen consumption. However, a point is reached where exercise intensity can continue to increase without the associated rise in oxygen consumption. At the point which oxygen consumption plateaus defines the $\mathrm{VO}_{2}$ max or an individual's maximal aerobic capacity. It is generally considered the best indicator of cardiorespiratory endurance and aerobic fitness. However, it is more useful as an indicator of a person's aerobic potential or upper limit than as a predictor of success in endurance events.

$\mathrm{VO}_{2}$ max is usually expressed relative to bodyweight because oxygen and energy needs differ relative to size. It can also be expressed relative to body surface area and this may be a more accurate when comparing children and oxygen uptake between sexes.

\section{OBJECTIVES}

1. To record physical anthropometric measurements of young female athletes of Mandya district in South Karnataka.

2. To record the $\mathrm{VO}_{2}$ max with particular reference to cardio-respiratory function in young female athletes during three phases of menstrual cycle by making use of Harvard step test. 
3. The subjects monitored their Basal body temperature (BBT) for 2 months prior to the study to determine their regular menstrual cycle.

4. To draw a comparison between three phases of menstrual cycle in this study and with the data available from other parts of the country.

SUBJECTS AND METHODS: $\mathrm{VO}_{2}$ max can be determined through a number of physical evaluations. These tests can be direct or indirect. Direct testing requires sophisticated equipment to measure the volume and gas concentrations of inspired and expired air. There are many protocols used on treadmills, cycle ergometer and other exercise equipment to measure $\mathrm{VO}_{2}$ max directly.

One of the most common is the Bruce protocol, often used for testing $\mathrm{VO}_{2}$ max in athletes or for signs of coronary heart disease in high risk individuals.

Indirect testing is much more widely used by coaches as it requires little or no expensive equipment.

VO $_{2}$ MAX TESTS: These are the tests for maximal aerobic power. $\mathrm{VO}_{2}$ max is the first choice in measuring to assess a person's cardiorespiratory fitness.(4) It is a fundamental measure of physiologic functional capacity for exercise. $(5,6,7)$

METHODS OF DIRECTLY ASSESSING AEROBIC POWER: There are three general methods of appraising maximal oxygen consumption.

1. Treadmill (running and walking)

2. Cycling (bicycle ergometer)

3. Stepping (step bench)

The manner in which the workload can be increased in these tests is either discontinuous or continuous. Expired air is collected in a Douglas bag and analysed for oxygen content.

METHODS OF INDIRECTLY ASSESSING AEROBIC POWER: Tests for direct assessment of $\mathrm{VO}_{2}$ max is limited in that the test is difficult, exhausting and often hazardous. For this reason, several indirect methods for predicting $\mathrm{VO}_{2}$ max from submaximal exercise data have been developed.

1) Astrand - Asrand Nomogram(8)

This was originally constructed from data gathered on young (18 to 30 years), healthy, physical education students, and it is based on the idea that heart rate during submaximal exercise increases approximately linearly with oxygen uptake and the nomogram was said to be more accurate if heart rates between 125 and 170 beats per minute were used to make predictions of $\mathrm{VO}_{2}$ max. For subjects older than 25 years, age correction factors must be used.

THE FOX EQUATION (9): This is a simple method for predicting $\mathrm{VO}_{2}$ max in males. It is based on a linear equation relating the directly measured $\mathrm{VO}_{2}$ max to the submaximal heart rate (HR sub) response.

The equation is:

Predicted $\mathrm{VO}_{2} \max (\mathrm{lit} / \mathrm{min})=6.3-(0.0193 \times \mathrm{HR}$ sub $)$ 
The standard error of the method for prediction of $\mathrm{VO}_{2}$ max from submaximal exercise test is about 10 percent in relatively well trained individuals of the same age and when employed as a screening test a consistent difference between measured and predicted maximal oxygen uptake of a few $100 \mathrm{ml} / \mathrm{min}$ is of no importance.

PREDICTIONS FROM NON-EXERCISE DATA (10): A unique approach to $\mathrm{VO}_{2}$ max prediction for quick screening of large group of individuals involves collecting specific non-exercise data from a questionnaire.

Data input to predict $\mathrm{VO}_{2} \max$

1. $\operatorname{Sex}($ Female $=0 ;$ Male $=1)$

2. BMI

3. Physical activity rating (PA-R). A point value between 0 and 10 representing overall physical activity level for the previous 6 months.

4. Perceived functional ability (PFA). Sum of the point values between 0 and 13 for questions about current level of perceived functional ability to maintain a continuous pace to cover a distance of 3 miles without becoming breathless or overly fatigued.

The equation is:

$\mathrm{VO}_{2} \max (\mathrm{ml} / \mathrm{kg} / \mathrm{min})=$ $44.895+(7.042 \times$ sex $)-(0.823 \times$ BMI $)+(0.738 \times \mathrm{PFA})+(0.688 \times \mathrm{PA}-\mathrm{R})$

Ethical clearance for the study protocol was obtained from the institute ethical committee.30 healthy female athletes of age group 17-20 years were selected by simple random method from a group of participants.

Informed consent was obtained from each subject. Those subjects with history of regular menstrual cycle were included. Subjects with history of menorrhagia, any gynaecological problems, who are taking oral contraceptives or any other medications, who smoked and consumed alcohol were excluded from the study.

Thus the health of the subject was assessed by noting the present, past, family and personal history and also by a thorough general and systemic examination.

The subjects were explained about the importance and procedure of the study. The subjects were asked not to change their life style during the period of study.

All the exercise data was collected from 7am to 9am. Data on physical characteristics such as age, height using stadiometer, weight using weighing scale and body mass index (BMI) was obtained. BMI was calculated as weight $(\mathrm{kg}) /$ height $(\mathrm{m})^{2}$.

The study involved non-invasive procedures with no financial burden on the subjects. The details of the procedure of exercise test were explained to the subjects and actually demonstrated before in order to allay apprehension.

In the present study, $\mathrm{VO}_{2}$ max was indirectly assessed by the Astrand nomogram method from submaximal exercise data obtained using Harvard step test.

Maximal heart rate is counted after each subject completed 'up' and 'down' task (22 steps per min) on a 16 inch bench (Harvard step bench) for 3 minutes until exhaustion whichever is early. 
The astrand nomogram (fig - 1), maximal heart rate and maximal oxygen consumption reached are connected in the nomogram. $\mathrm{VO}_{2} \max \left(\mathrm{in} \mathrm{lit} / \mathrm{min}\right.$ ) is read from the $\mathrm{VO}_{2}$ scale. The corresponding values of $\mathrm{VO}_{2}$ max in terms of bodyweight are calculated.

Since the subjects in this study did not exceed 25 years of age, age correction factor was not applied.

$\mathrm{VO}_{2}$ max recordings were made on specified days in the menstrual cycle in 3 phases, menstrual phase - from $1^{\text {st }}$ to $5^{\text {th }}$ day of the cycle, follicular phase - from $8^{\text {th }}$ to $12^{\text {th }}$ day of the cycle, luteal phase - from $20^{\text {th }}$ to $25^{\text {th }}$ day of the cycle and this was indirectly assessed by the Astrand nomogram method from submaximal exercise data obtained using Harvard step test.

STATISTICAL ANALYSIS AND RESULTS: All the statistical operations were done through SPSS for Windows (Version 15 evaluation Version, 2006), SPSS Inc. New York (Statistical presentation system software)

In the present study, $\mathrm{VO}_{2}$ max during follicular, luteal and menstrual phases of the menstrual cycle is noted. The difference in the mean values between the three phases of the menstrual cycle was determined by using student' $t$ ' test. The calculated value was compared with the table value for corresponding degree of freedom at 0.05 level of significance. Thus $\mathrm{P}>0.05$ was considered not significant and $\mathrm{P}<0.05$ was considered significant.

ANTHROPOMETRIC MEASUREMENTS: The participants recruited were almost of the same height, weight, age and BMI without exhibiting a statistically significant difference. (Table - 1)

\section{TABLE - 1}

\begin{tabular}{|l|l|l|}
\hline & Mean & S.D \\
\hline Age (yrs) & 18.07 & 0.94 \\
\hline Height $(\mathrm{cms})$ & 162.70 & 5.38 \\
\hline Weight $(\mathrm{kgs})$ & 50.70 & 3.48 \\
\hline BMI $\left(\mathrm{kg} / \mathrm{m}^{2}\right)$ & 19.13 & 1.45 \\
\hline
\end{tabular}

$\mathbf{V O}_{2}$ MAX: Comparison of changes in the $\mathrm{VO}_{2}$ max during follicular, luteal and menstrual phases of the menstrual cycle showed statistically no significant changes. (Table -2 and Table -3 )

TABLE - 2: Showing changes in the $\mathrm{VO} 2 \mathrm{max}$ in $\mathrm{ml} / \mathrm{kg} / \mathrm{min}$ during three phases of menstrual cycle of all subjects.

\begin{tabular}{|l|l|l|}
\hline V02 max (ml/kg/min) & Mean & S.D \\
\hline Follicular(1) & 51.72 & 5.43 \\
\hline Luteal(2) & 50.23 & 5.48 \\
\hline Menstrual(3) & 49.24 & 4.77 \\
\hline
\end{tabular}

TABLE - 3: Comparison of changes in the $\mathrm{VO2}$ max in $\mathrm{ml} / \mathrm{kg} / \mathrm{min}$ during three phases of menstrual cycle of all subjects.

\begin{tabular}{|l|l|}
\hline Follicular(1)Vs Luteal(2) & 0.165 (P value) \\
\hline
\end{tabular}




\section{ORIGINAL ARTICLE}

\begin{tabular}{|l|l|}
\hline Follicular(1)Vs Menstrual(3) & 0.856 (P value) \\
\hline Luteal(2)Vs Menstrual(3) & 0.882 (P value) \\
\hline
\end{tabular}

DISCUSSION: The typical menstrual cycle is of 28 days and first day of the menstrual bleeding considered to be the first day of menstrual cycle, followed by the proliferative phase from $6^{\text {th }}$ to $14^{\text {th }}$ day, then secretory phase is from $15^{\text {th }}$ to $28^{\text {th }}$ day. Ovulation usually occurs 14 days before the beginning of next menstrual cycle and this can be best predicted by Basal body temperature (BBT), Cervical smear, Endometrial biopsy, Serum progesterone estimation and Urinary LH measurement. In the present study BBT is used to predict the ovulation.

During the course of menstrual cycle some predictable and measurable variations in the concentration of female sex hormones exists i.e., Estrogen and Progesterone. This change in hormonal pattern will have an impact on athletic performance. In turn athletic performance also depends upon physiological, biochemical and psychological factors. The main physiological factor includes cardiovascular changes and respiratory changes.

During the proliferative phase the important hormones are estrogen and progesterone. As the estrogen concentration is higher than progesterone, this phase is also known as estrogen phase. Estrogen is secreted primarily by the granulosa cells of the ovarian follicle. The level of estradiol secretion rate is $36 \mu \mathrm{g} / \mathrm{d}$ in the early follicular phase and reaches a peak level of $380 \mu \mathrm{g} / \mathrm{d}$ just before ovulation and $250 \mu \mathrm{g} / \mathrm{d}$ during the mid-luteal phase.(11) The estrogen apart from reproductive function has many beneficial effects on the body such as cardiovascular system, bone, brain and the substrate metabolism. The main function on the cardiovascular system includes increase in the capillary wall strength, vasodilatory effect on vascular smooth muscle in coronary arteries and peripheral vascular beds. In addition it also lowers the cardiovascular response to stress. (12)

The present study conducted on young female athletes during follicular phase of the menstrual cycle, $\mathrm{VO}_{2}$ max showed no statistically significant changes. The mean value and S.D. of $\mathrm{VO}_{2}$ max during this phase is $51.72 \pm 5.43 \mathrm{ml} / \mathrm{kg} / \mathrm{min}$. Therefore, the beneficial effect of estrogen during proliferative phase of menstrual cycle has no significant effect on $\mathrm{VO}_{2}$ max.

During the luteal phase the important hormones are estrogen and progesterone. As the progesterone concentration is higher than estrogen, this phase is also known as progestational phase. Progesterone is secreted by the corpus luteum and in small amounts in the follicle. During the early follicular phase, the progesterone level is $0.9 \mathrm{ng} / \mathrm{ml}$, steadily increases in the late follicular phase and then attains a peak value of approximately $18 \mathrm{ng} / \mathrm{ml}$ in the late luteal phase.(11) Progesterone is known to affect thermoregulation, ventilation and substrate metabolism. Higher progesterone level in the luteal phase acts directly on the medullary respiratory center where it lowers the threshold level and also increases its excitability resulting in greater minute ventilation and maximal exercise response. Thereby in untrained athletes this has shown to be significant especially who experience subjective dyspnoea whereas in trained athletes no correlation actually exists between ventilation and progesterone levels. (12)

The present study conducted on young female athletes during luteal phases of the menstrual cycle to assess $\mathrm{VO}_{2}$ max showed no statistically significant changes. The mean value and S.D. of $\mathrm{VO}_{2}$ max during this phase is $50.23 \pm 5.48 \mathrm{ml} / \mathrm{kg} / \mathrm{min}$.

During the menstrual phase, the estrogen and progesterone levels are decreased especially progesterone at the end of the ovarian cycle. This phase has no beneficial effect either on 
cardiovascular system or respiratory system. During this phase, $\mathrm{VO}_{2}$ max, showed no statistically significant changes. . The mean value and S.D. of $\mathrm{VO}_{2}$ max during this phase is $49.24 \pm 4.77 \mathrm{ml} / \mathrm{kg} / \mathrm{min}$.

The sex hormones estrogen and progesterone with varying hormone levels of concentration during different phases of the menstrual cycle have complex interactions on the body. The net physiological effects of both the hormones can be either opposing or synergistic to bring about the beneficial effects on the body.(12)

In the present study, the net effect of estrogen and progesterone on $\mathrm{VO}_{2}$ max measured by using Astrand nomogram during follicular, luteal and menstrual phases showed no statistically significant changes. Our results are consistent with study by Pratima Chatterjee et al.(13) who reported there was no statistically significant changes in $\mathrm{VO}_{2}$ max during the different phases of menstrual cycle. Our results is also consistent with study by Teresa M. Dean, et al.(14)who reported that $\mathrm{VO}_{2}$ max, maximal heart rate and other measures of cardio-respiratory fitness are not significantly affected by the changing sex steroid levels observed across the normal menstrual cycle.

CONCLUSION: In our study, $\mathrm{VO}_{2}$ max showed statistically no significant changes during all the three phases of menstrual cycle. This suggests that regularly menstruating female athletes competing in strength specific sports need not adjust for menstrual cycle phase to maximize performance.

LIMITATIONS OF THE STUDY: The limitations of the present study are less sample size and lack of measurement of other cardio-respiratory fitness parameters.

\section{REFERENCES}

1. Shephard RJ. Exercise and training in women, Part 1 : Influence of gender on exercise and training responses. Can J Appl Physiol 2000; 25: 19-34.

2. Shephard RJ. Exercise and training in women, Part 11: Influence of menstrual cycle and pregnancy on exercise responses. Can J Appl Physiol 2000; 25: 35-54.

3. Wilmore JH, Costill DL. Physiology of Sport and Exercise. $3^{\text {rd }}$ edition, Champaign, IL : Human Kinetics. 2005.

4. Fox E. Difference in metabolic alterations with sprint versus endurance interval training. Metabolic Adaptation to prolonged physical exercise, Basel, Switzerland : BirkhauserVeralag. 1975; p. 119-126.

5. Mc Ardle WD, et al. Reliability and inter-relationships between maximal oxygen uptake, physical work capacity and step scores in college women. Med Sci Sport 1972; 4: 182.

6. Mc Ardle WD, et al. Exercise physiology. $5^{\text {th }}$ edition, 2001; p. 231-248.

7. Taylor HL, et al. Maximal oxygen uptake as an objective measure of the cardio-respiratory performance. J ApplPhysiol 1955; 8: 73-80.

8. Astrand P, Rhyming I. A nomogram for calculation of aerobic capacity (physical fitness) from pulse rate during submaximal work. J ApplPhysiol 1954; 7: 215-221.

9. Fox E. A simple, accurate technique for predicting maximal aerobic power. J ApplPhysiol 1973; 35(6): 914-916. 


\section{ORIGINAL ARTICLE}

10. George JD, et al. Non-exercise $\mathrm{VO}_{2}$ max estimation for physically active college students. Med Sci Sports Exerc 1997; 29: 415.

11. William F. Ganong. Review of Medical Physiology. 22nd edition, McGraw Hill, Lange Medical publications, Singapore, 2005; p.440-443.

12. Lebrun CM, Rumball JS. Relationship between athletic performance and menstrual cycle. Current Women's Health Reports 2001; 1(3): 232-240.

13. Pratima Chatterjee, Samir Nag, Minati Kali, Chatterjee S. Study on VO2 max of female athletes in relation to menstrual cycle. Ind J Physiol and Allied Sci 1988; 42(1): 11-13.

14. Teresa M. Dean, Leigh Perreault, Robert S. Mazzeo, Tracy J. Horton. No effect of menstrual cycle phase on lactate threshold. J ApplPhysiol 2003; 95: 2537-2543. 


\section{ORIGINAL ARTICLE}

Graph - 1: Comparison of changes in the $\mathrm{VO}_{2} \max$ in $\mathrm{ml} / \mathrm{kg} / \mathrm{min}$ : Follicular, Luteal and Menstrual phases.
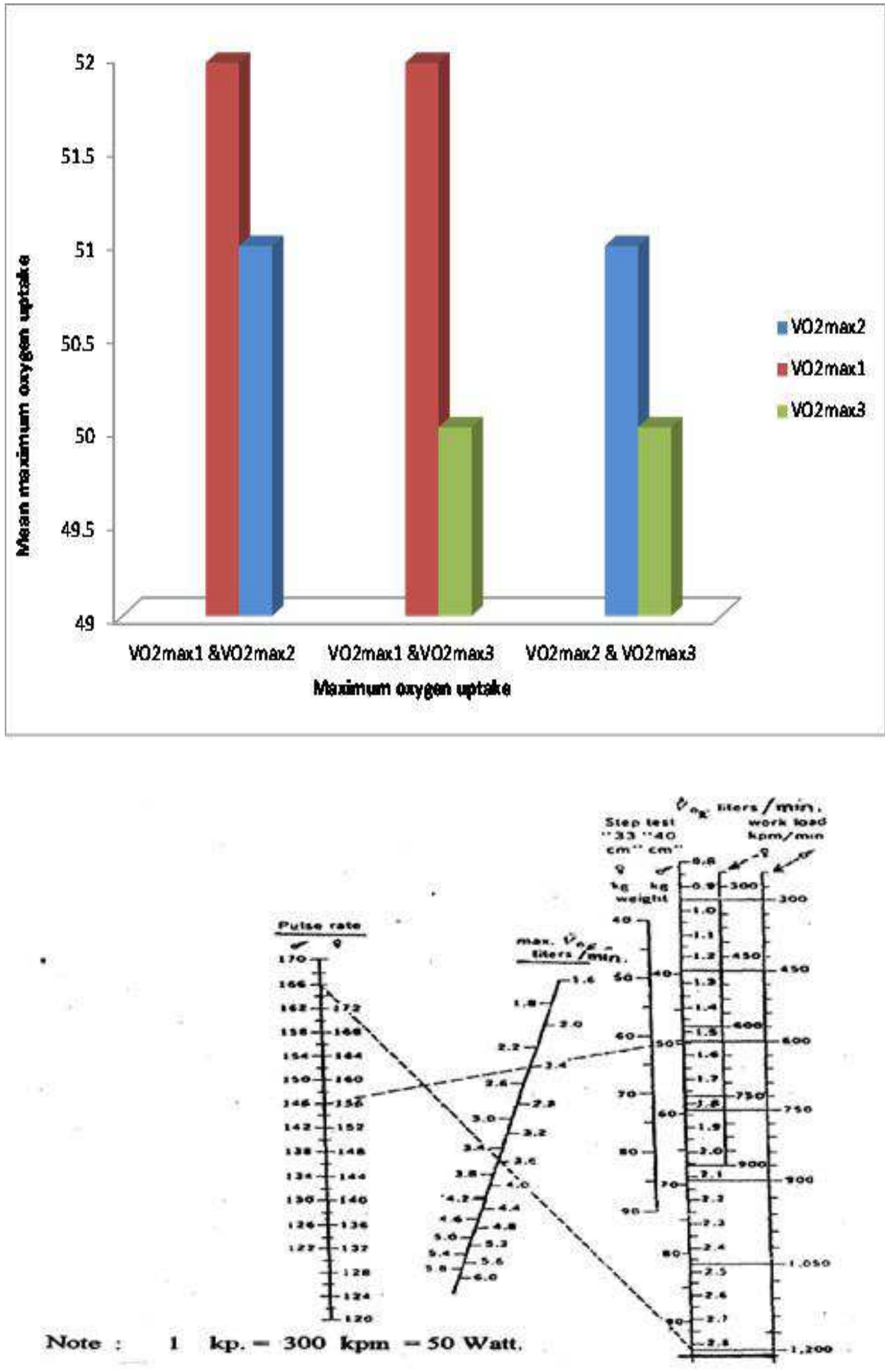

FIG 1: Astrand nomogram 